\title{
From preformation to reformulation-the then and now of sperm selection
}

\author{
David F. Albertini ${ }^{1}$
}

Published online: 30 September 2017

(C) Springer Science+Business Media, LLC 2017

As much as we have learned about the intratesticular, extratesticular, and intrauterine lifespan of human sperm, sorting out those capable of enacting the first steps in human development remains a major challenge in ARTs. From their very beginnings as stem cells transformed into primary spermatocytes (see issue cover), these highly differentiated cells stored in the epididymis assume an identity and character conducive to fertilization and egg activation only after receiving an appropriate set of cues from the environments they transit.

While the cargo transmitted by sperm to the nascent zygote is being clarified as an ever-growing list of long sought consumables (see below), putting the male gamete on equal footing with that of the female has for years spawned a debate extending well back into the sixteenth century. Think Homunculus. In her scholarly work of 1997, Clara PintoCorreira (The Ovary of Eve: Egg and Sperm and Preformation, ISBN 0-226-66952-1, University of Chicago Press) traces the tortuous debate that raged in the quest for understanding the very roots of embryology when (real data aside) the guesswork of the spermists and ovists sought truth to their respective claims of life having its origins in one but not the other of sexes. In its various iterations, the battle of the sexes continues today as reproductive medicine uneasily basks in a scholarly medium rich in molecular and mechanistic detail comparable to the legendary battle waged in the 1970s between Billie Jean King and the late Bobby Riggs. Bragging rights aside, until recently the ovists have domi-

David F. Albertini

eicjarg@gmail.com

1 The Center for Human Reproduction, New York, NY, USA nated the gamete landscape in the arena of human ARTs, much as it became appreciated in yesteryear upon the advent of microscopy - when seeing became believing.

With this back drop, we deliver our readership a series of articles highlighting in a tempered and tempting fashion of some of the issues arising from a more spermatocentric orientation. From understanding and managing the risks associated with sperm-borne viral transmission, Dubaut and colleagues provide a timely update on Zika infections (Impact of Zika virus for infertility specialists: current literature, guidelines, and resources https://doi.org/10.1007/s10815-017-0988) through to new and old genetic discoveries that are adding to our understanding of the multidimensional nature of male fertility and infertility (The -29 G/A FSH receptor gene polymorphism is associated with higher FSH and LH levels in normozoospermic men https://doi.org/10.1007/s10815017-0970; Detection of candidate nectin gene mutations in infertile men with severe teratospermia https://doi.org/10. 1007/s10815-017-0985-4).

In practical terms, the introduction and application of cryopreservation to ARTs were milestones initially staged for animal semen and readily translated into sperm banking for humans, paving the way for the "lay away" mentality that is so pervasive and prominent in reproductive medicine. Towards this end, Palomar Rios and colleagues draw attention to some of the limitations of sperm cryopreservation as we know them today and suggest directions for future improvements likely to make a difference in fertility preservation strategies for men (Sperm parameters that play a major role in the assessment of semen quality after cryopreservation https://doi. org/10.1007/s10815-017-0973-8). And teasing apart causes of male infertility relative to other conditions such as metabolic syndrome has proven to be a serious challenge warranting the search for alternative biomarkers of male reproductive health (Serum microRNAs in male subfertility_-biomarkers 
and a potential pathogenetic link to metabolic syndrome https://doi.org/10.1007/s10815-017-0989).

Seeking out the causes of male infertility presently commands much attention. While formative research in animal models has contributed much in terms of genetic and mechanistic insights [1], when it comes to practical applications like discerning and manipulating well-seasoned processes like capacitation, there remains little consensus as to how to proceed to fill such an important gap in basic biology [2]. Exemplary of problems where the basic science has lagged well behind the need for clinical manipulation is the case of PLCzeta and egg activation. In JARG and elsewhere, we have seen repeated efforts to deploy calcium ionophores clinically to overcome the apparent resistance of oocytes from some patients to undergo activation after ICSI or IVF. While buttressed by the now firmly established documentation of sperm-borne PLCzeta as a key player in the initiation of calcium transients required for egg activation in mice, recent research suggests more complexity exists [3]. Time will tell just how far away we are for negotiating the subtleties of human fertilization.

Stepping back from sperm function in egg activation is the more proximate matter of how did they get there in the first place. Slithering through the forest of egg coat materialsknown affectionately to the human ART community as the zona pellucida - is not simply a case of sink or swim! Rather, reports on the molecular structure of egg coat materials, including the mammalian zona pellucida component $\mathrm{ZP} 2$, have now revealed the presence of a sophisticated and apparently evolutionarily conserved mechanism for sperm penetration [4]. The proposed gating mechanism involves interaction of sperm lysins with ZP2 effecting creation of pores through which motile sperm can pass. We can begin to imagine a time where drugs designed to enhance the pore-forming activity in the zona may be coupled with motility increasing compounds like myoinositol [5], an interventional strategy already being inserted into clinical practice.

The real question emerging from studies of human sperm is whether or not we should be taking these remedial efforts to intervene with presumed deficits, when direct evidence is lacking?
It was only a year ago that sperm RNA cargo was placed center stage as a potential modifier of embryo development, a situation now being revisited in view of possible contamination in the semen preparations used for that study [6]. Whatever cargo human sperm is eventually shown to contain, increasing concerns that perturbations at the epigenetic level underscore the disturbing trend towards oligospermia all too evident in industrialized countries of the world are to be taken to heart as ARTs move forward [7]. It is in the realm of now that our abilities to select sperm capable of propagating healthy offspring must be reckoned with. And what about back then? Back in the testis, when spermatogonial stem cells complete mitosis and foster the onset of meiosis. Sealing the deal for making healthy babies starts early. The fact that exposures of males to environmental estrogens pose long-term effects in later generations is becoming an all too familiar message in the recent literature - a situation far too precarious to ignore in the realm of human ARTs...then and now!

\section{References}

1. Coutton C, Fissore RA, Palermo GD, Stouffs K, Toure A. Male infertility: genetics, mechanism, and therapies. Biomed Res Int. 2016;2016:7372362.

2. De Jonge C. Biological basis for human capacitation-revisited. Hum Reprod Update. 2017;23(3):289-99.

3. Hachem A, Godwin J, Ruas M, Lee HC, Ferrer Buitrago M, Ardestani G, et al. PLCzeta is the physiological trigger of the $\mathrm{Ca} 2+$ oscillations that induce embryogenesis in mammals but conception can occur in its absence. Development. 2017;144(16):2914-24.

4. Raj I, Sadat Al Hosseini H, Dioguardi E, Nishimura K, Han L, Villa A, et al. Structural basis of egg coat-sperm recognition at fertilization. Cell. 2017;169(7):1315-26. e17

5. Condorelli RA, La Vignera S, Mongioi LM, Vitale SG, Lagana AS, Cimino L, et al. Myo-inositol as a male fertility molecule: speed them up! Eur Rev Med Pharmacol Sci. 2017;21(2 Suppl):30-5.

6. Cappallo-Obermann H, Spiess AN. Comment on "absence of sperm RNA elements correlates with idiopathic male infertility”. Sci Trans1 Med. 2016;8(353):353tc1.

7. Kobayashi N, Miyauchi N, Tatsuta N, Kitamura A, Okae H, Hiura H, et al. Factors associated with aberrant imprint methylation and oligozoospermia. Sci Rep. 2017;7:42336. 